design of work procedures by and for health and social care teams.

Methods Over a 3-week period in April 2020, 66 international clinicians, managers, medical scientists, improvement advisors and human factors experts routinely met virtually online to identify and agree on basic guiding principles to inform the user-centred design of work procedures. Pragmatic consensus building based on a modified-Delphi approach was used.

Results Ten key guidance steps were identified and agreed upon to assist health and social care teams in how to design work procedures that were usable, safe and effective e.g., involve the whole team; identify the hazards; capture workas-done; make it easy to follow; and keep it under review (figure 1).

Conclusions For a work procedure to be fully accepted and used, the relevant care team should be involved from the start and throughout the design and implementation process. The developed guidance outlines practical pointers for all care teams in any setting worldwide that are conducive to good design practice in this area.

\section{IMPROVING ADOLESCENT HIV TEST COMPLETION IN THE CLINICAL SETTING}

Debra Braun-Courville. Vanderbilt University Medical Center

\section{$10.1136 / \mathrm{bmjoq}-2021-\mathrm{IHI} .2$}

Background In 2018, 26\% of new HIV diagnoses in the US were among youth 13-24 years of age; more than 50\% had undiagnosed infections and were unaware they had HIV. Routine screening and targeted HIV testing is recommended for adolescents. Providers at the Adolescent and Young Adult Clinic at Vanderbilt order HIV testing based on standardized guidelines and clinical indicators, but completion of the laboratory test is not universal.

Objectives To improve the percentage of completed HIV tests within 1 week of ordering from $71.4 \%$ to $>95 \%$ in the clinical setting by June 30, 2019.

Methods Interventions were tested and implemented over an 8 -month period based on a key driver diagram created by the improvement team (figure 1). The primary outcome was percentage of adolescents aged 12 to 22 years who had HIV testing ordered and successfully completed the test within one

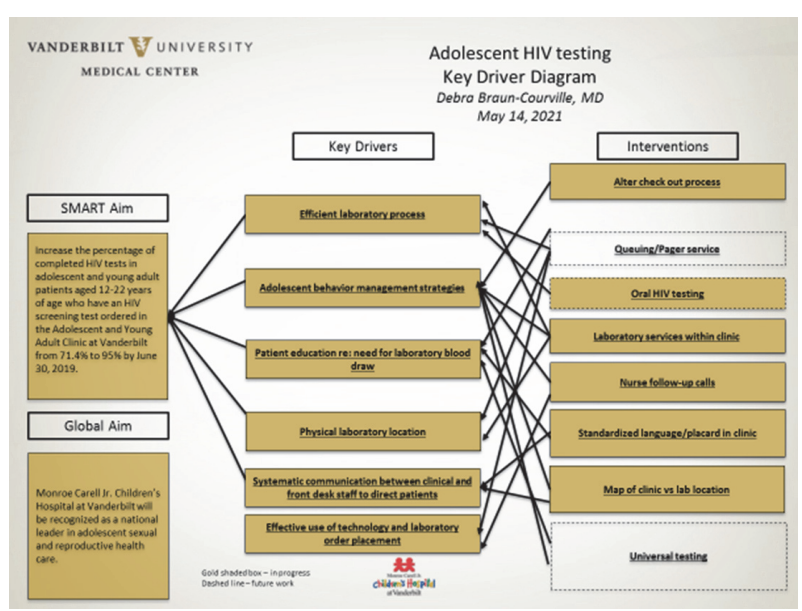

Abstract 2 Figure 1 Key driver diagram

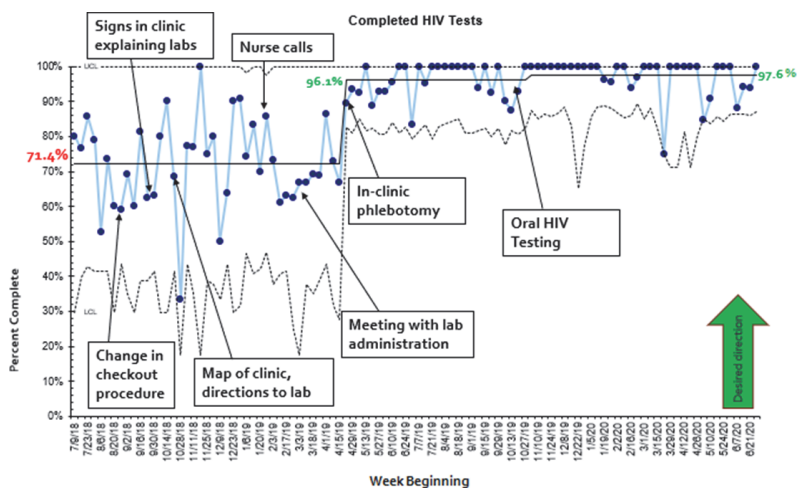

Abstract 2 Figure 2 Control chart

week of order entry. Data was followed prospectively following the initiation of the project. Statistical process control charts were used to view and analyze data with special cause variation identified using Nelson rules.

Results $71.4 \%$ of patients completed the ordered HIV test at baseline (July-September 2018). Adolescent-specific educational and behavioral modification strategies were tested to improve laboratory completion rates. Provision of in-clinic phlebotomy services as well as oral HIV testing facilitated project success. After several plan-do-study-act cycles, which included behavioral interventions as well as clinic-wide system modifications, special cause variation was achieved and $96.1 \%$ of patients completed HIV testing within 1 week of order entry. This completion rate has been successfully maintained for more than 12 months post-interventions (figure 2).

Conclusions Educational interventions in the absence of robust behavioral modification tools may have limited availability in practice. Data can drive patient improved outcomes and allow teams to expand in-clinic services to improve the delivery of care.

\section{A NOVEL APPROACH TO SETTING GOALS TO ACHIEVE A REDUCTION IN ACUTE-CARE LENGTH OF STAY}

Amy D Bell, Ed Dunlow, Gary Little. Atrium Health

\subsection{6/bmjoq-2021-|HI.3}

Background Length of stay (LOS) can be used to measure cost savings when expressed in terms of opportunity days. Using simple tools to assess the percent of contribution for opportunity days by facility and service line, along with a financial target and standard proxy cost per day, LOS goals can be established for the number of opportunity days to save for each facility and service line within a healthcare system.

Objectives Utilize percent of opportunity day contributions to set acute care facility and service line targets based upon a financial goal for a healthcare system.

Methods Targets for LOS opportunity days to save are determined based upon the proportion of the financial goal each facility is expected to contribute to the overall cost savings. An opportunity day is defined as the difference between the number of days a patient stays in the hospital versus the number of days they were expected to stay, determined by Care Science risk adjustment (figure 1). Opportunity days to be saved were translated into an Observed/Expected (O/E) Ratio target for each facility (figure 2). 
Results LOS reduction efforts resulted in days saved in nonCOVID patients, but the negative savings from COVID patients resulted in a net loss of opportunity days when all patients were bundled together (figure 3). Calculations for opportunity days saved were modified to account for the decrease of inpatient volumes and subsequent analysis of the

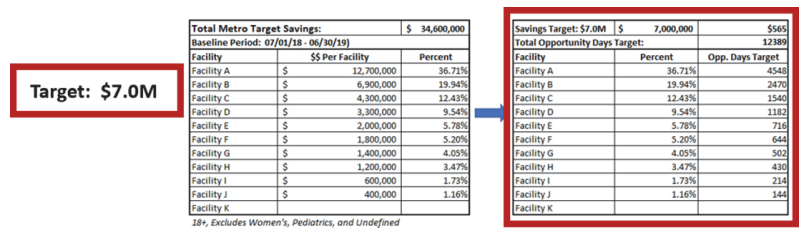

Abstract 3 Figure 1

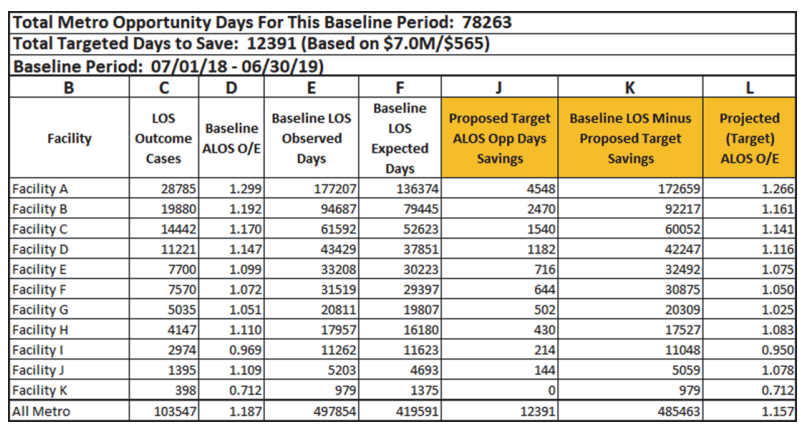

Abstract 3 Figure 2

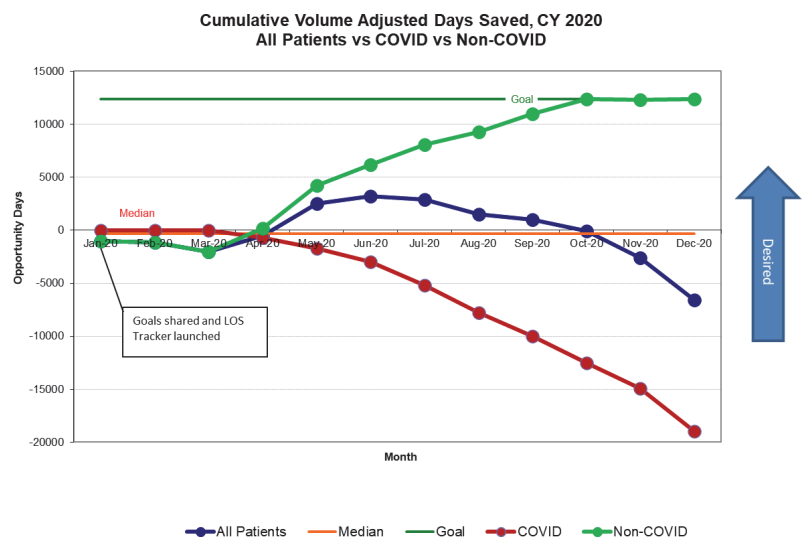

Abstract 3 Figure 3

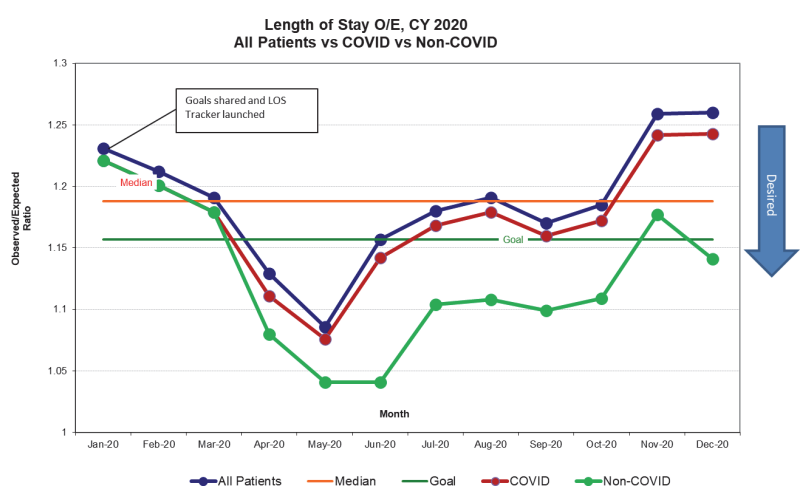

Abstract 3 Figure 4 data included stratification for the COVID vs. non-COVID population.

Conclusions The model described for combining a financial goal by facility with opportunity days enables the setting of LOS targets for healthcare systems. Further, the model supports tracking progress to targets, including the ability to compare specific patient types, e.g., COVID-19 Positive vs. COVID-19 Negative (figure 4).

\section{RIGHT CARE AT THE RIGHT TIME: A CARE MODEL REDESIGN QUALITY IMPROVEMENT PROJECT}

William Bekker, Caitlin Garrett, Heather Gleason, Kathryn Hoehn, Katie Iommazzo, Karen Lane, Benji Mathews, Hilary Radtke, Sara Spilseth, Julie Weegman. Regions Hospital

\subsection{6/bmjoq-2021-|HI.4}

Background Over the last several years, there have been focused efforts to continually improve communication amongst the care team while maintaining patient and family centered care, progressing the patient through their care as clinically indicated, and ensuring timely and efficient discharge planning. Despite these efforts, there was a lack of reliability in the care model which created gaps amongst care team members (inclusive of the patient) in the knowledge of the plan of care.

Objectives Care Model redesign is an approach aimed to decrease overall length of stay, improves workflow efficiencies that prioritize quality and timely care, and set a "gold standard" for hospital patient care. The goal of the inpatient care model was to improve both patient and staff satisfaction, while ensuring processes that support patient throughput and overall access to care.

Methods The Model for Improvement, best practice review, adaptive change management, human centered design tools, and PDSA cycles were utilized within the project. We created a new care model with structured connections and tools to support discussions. We engaged front line staff through a design session and optimized current technology to merge segmented work into one cohesive approach.

The new care model was initially piloted, evaluated, and spread to eight units across the hospital. The team leveraged a multidisciplinary coaching model, and consistent leadership presence to ensure engagement of all team members.

Results The data highlights reduced variation by creating a more reliable model to replicate. This resulted in a $21 \%$ rate of improvement, and we have exceeded the organization's goal of $20 \%$ of discharges by 11:00am (figure 1). The team identified process, outcome, and balancing metrics for this project that were closely monitored and reviewed (figure 2).

Conclusions There is a continued commitment to follow the new process 7 days a week, identify opportunities and

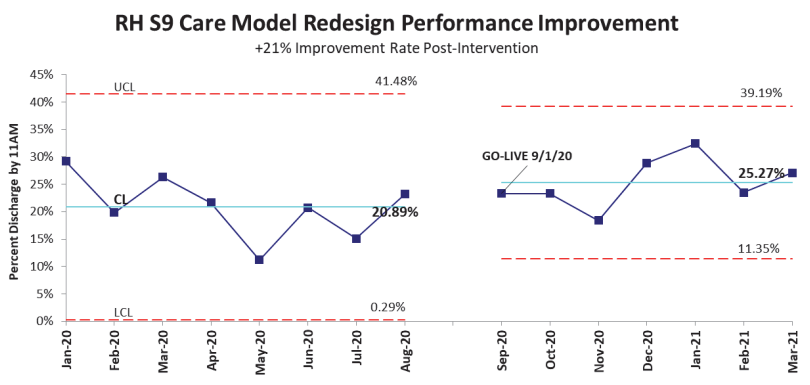

Abstract 4 Figure 1 